Fetal Diagnosis and Therapy

\title{
Embryo Reduction in Dichorionic Triplets to Dichorionic Twins by Intrafetal Laser
}

\author{
Petya Chaveeva ${ }^{a}$ Przemek Kosinski $^{a}$ Cahit Birdir $^{a} \quad$ Laszlo Orosz $^{a}$ \\ Kypros H. Nicolaides ${ }^{a, b}$ \\ ${ }^{a}$ Harris Birthright Research Centre for Fetal Medicine, King's College Hospital, and ${ }^{b}$ Department of Fetal Medicine, \\ University College Hospital, London, UK
}

\section{Key Words}

Triplet pregnancy · Embryo reduction - Laser surgery · First trimester $\cdot$ Miscarriage $\cdot$ Preterm birth

\begin{abstract}
Objective: To describe a new technique for embryo reduction (ER) in dichorionic triplet (DCT) pregnancies. Methods: In 22 DCT pregnancies, ER to dichorionic twins was carried out at 11.3-13.9 weeks' gestation by ultrasound-guided laser ablation of the pelvic vessels of one of the monochorionic twins. Results: Intrafetal laser was successfully carried out in all cases, but ultrasound examination within 2 weeks of the procedure demonstrated that the co-twin had died in 11 cases and was alive in the other 11 . In the dichorionic group there was one miscarriage at 23 weeks due to cervical incompetence and in the other 10 cases there were two live births at a median gestational age of 35.0 (range 32.2-37.1) weeks. In the 11 cases where both monochorionic fetuses died the separate triplet was live born at a median gestation of 38.0 (range 32.2-40.5) weeks. Conclusions: In the management of DCT pregnancies, ER to dichorionic twins by intrafetal laser is an additional option to the traditional ones of expectant management, ER by intrafetal injection of potassium chloride $(\mathrm{KCl})$ to monochorionic twins or $\mathrm{ER}$ by $\mathrm{KCl}$ to singleton.

(c) 2013 S. Karger AG, Basel
\end{abstract}

(c) 2013 S. Karger AG, Basel

$1015-3837 / 13 / 0352-0083 \$ 38.00 / 0$

\section{Introduction}

In triplet pregnancies diagnosed during the first trimester management options include continuing with the whole pregnancy or embryo reduction (ER) to twins or singletons by fetal intracardiac injection of potassium chloride (KCl) [1]. In dichorionic triplet (DCT) pregnancies, ER involves either the dichorionic fetus or both monochorionic twins. The alternative of ER of one of the monochorionic twins is avoided because of the risk that the injected $\mathrm{KCl}$ could be transferred to the co-twin through the intertwin placental vascular anastomoses or death of one fetus could lead to haemorrhage from the co-twin into the dead fetoplacental unit with consequent death or neurodevelopmental impairment.

In this study we describe a new technique for ER in DCT pregnancies to twins, which involves the ultrasound-guided laser ablation of the pelvic vessels of one of the monochorionic twins.

\section{Methods}

This was part of a retrospective study of triplet pregnancies referred to our fetal medicine unit for counselling and further management between 1986 and 2013. In all cases included in the study ultrasound examination was carried out at 10-14 weeks' gestation to demonstrate three live fetuses, determine chorionicity from examination of the intertriplet membranes [2] and calculate gesta-

\section{KARGER}

E-Mail karger@karger.com www.karger.com/fdt
Prof. K.H. Nicolaides

Harris Birthright Research Centre for Fetal Medicine

King's College Hospital

Denmark Hill, London SE5 9RS (UK)

E-Mail kypros@fetalmedicine.com 
tional age from the crown-rump length of the biggest fetus [3]. Parents were counselled regarding the options of expectant management or ER. Maternal demographic characteristics, ultrasound findings and in those undergoing ER details of the procedure were recorded in a database. Pregnancy outcomes were collected into the same database when they became available from the referring hospitals, general practitioners or from the patients themselves.

In a previous study we reported the outcome of 528 trichorionic triplet pregnancies and 167 DCT pregnancies managed expectantly or by fetal intracardiac injection of $\mathrm{KCl}$ for $\mathrm{ER}$ to twins or singletons [1]. In this study we present the technique and outcome of 22 DCT pregnancies undergoing ultrasound-guided laser ablation of the pelvic vessels of one of the monochorionic twins.

Women received prophylactic antibiotics (cefuroxime, $750 \mathrm{mg}$, i.v.). Ultrasound examination was carried out to select the fetus for ER that could be accessed without traversing the monochorionic intertwin membrane. A transverse section of the lower fetal abdomen was obtained and colour flow Doppler was used to visualize the internal iliac arteries and intra-abdominal umbilical vein. Local anaesthesia (10 $\mathrm{ml} \mathrm{1 \%} \mathrm{lidocaine)} \mathrm{was} \mathrm{given} \mathrm{to} \mathrm{the} \mathrm{maternal} \mathrm{skin,}$ subcutaneous tissues and myometrium. An 18-gauge needle (Cook Ireland Ltd., Limerick, Ireland) was introduced and under continuous ultrasound visualization was guided to the fetal abdomen with the tip being adjacent to the pelvic vessels. A 400- $\mu$ m laser fibre was then inserted into the needle and advanced to a couple of millimetres beyond the tip of the needle. Laser coagulation was performed using NdYag laser (Dornier MedTech, Wessling, Germany) with $40 \mathrm{~W}$. This resulted, within a few seconds, in hyperechogenicity of tissues in the lower abdomen and cessation of blood flow in the iliac arteries and umbilical vein. Fetal heart activity continued for several minutes. After a period of rest for about $60 \mathrm{~min}$ another ultrasound examination was carried out to confirm death of one monochorionic twin and survival of a dichorionic pair. The patient was discharged home and a further appointment was given for an ultrasound examination within 2 weeks of the procedure.

\section{Results}

The study population of 22 DCT pregnancies undergoing ER to twins by intrafetal laser included 18 of Caucasian racial origin, 2 of Afro-Caribbean origin, 1 South Asian and 1 East Asian; 14 were nulliparous and 8 were parous, and conception was spontaneous in 4 and by in vitro fertilization in 18 . The median maternal age was 36.0 (range 19.1-45.6) years and the median gestational age at ER was $12.3(11.1-13.9)$ weeks.

Ultrasound examination had demonstrated that all three fetuses in each pregnancy were alive. There were no obvious defects in any of the separate fetuses. In the monochorionic twins, one fetus had diaphragmatic hernia, one had generalized oedema with nuchal translucency thickness of $12 \mathrm{~mm}$ and in 8 cases there were early signs of twin-to-twin transfusion syndrome or selective fetal growth restriction with large discordance in crown-rump length $(>10 \%)$ and subjectively assessed discordance in amniotic fluid volume and/or reversed a-wave in the ductus venosus in one of the fetuses.

At the end of the ER procedure and $1 \mathrm{~h}$ later both remaining dichorionic twins were alive and there was no blood flow within the dead fetus. However, ultrasound examination within 2 weeks after ER demonstrated that the monochorionic co-twin had also died in 11 cases (table 1). In the 11 cases where both monochorionic fetuses died the separate triplet was live born at a median gestational age of 38.0 (range 32.2-40.5) weeks. In the 11 cases with two surviving fetuses there was one miscarriage at 23 weeks' gestation due to cervical incompetence and the other 10 pregnancies resulted in two live births at a median gestational age of 35.0 (range 32.2-37.1) weeks. There were no cases of preterm prelabour amniorrhexis. None of the neonates had any obvious defects or evidence of neurological deficit.

In the total group, the rates of miscarriage before 24 weeks' gestation and preterm birth before 33 weeks were $4.5 \%$ ( 1 of 22 ) and $9.1 \%$ ( 2 of 22$)$.

\section{Discussion}

The findings of this study demonstrate that in the management of DCT pregnancies, ER to dichorionic twins by intrafetal laser is an additional option to the traditional ones of expectant management, ER by intrafetal injection of $\mathrm{KCl}$ to monochorionic twins or $\mathrm{ER}$ by $\mathrm{KCl}$ to singleton.

The technique of ER utilizing interstitial laser is similar to that used in interrupting the blood supply of the acardiac twin in twin reversed arterial perfusion sequence [46]. In our cases of DCT pregnancies treated by intrafetal laser although all monochorionic co-twins were alive $1 \mathrm{~h}$ after the procedure half of these twins died within the subsequent 2 weeks. The most likely explanation for the deaths was retrograde haemorrhage into the placenta of the dead fetus or the fetus itself if the occlusion of the fetal pelvic vessels was incomplete. In a total of 16 cases of twin reversed arterial perfusion sequence treated by intrafetal laser at 12-20 (median 16) weeks' gestation there was death of the pump twin within 2 weeks of the procedure in 5 (31\%), which was attributed to incomplete vascular occlusion and haemorrhage into the dead acardiac twin $[5,6]$.

In $82 \%$ of our DCT pregnancies conception was by in vitro fertilization. The risk of monozygotic twinning in assisted conception is more than twice as high as the $0.4 \%$ rate in natural conceptions $[7,8]$. Although the overall rate of in vitro fertilization-related multiple pregnancies 
Table 1. Outcome of DCT pregnancies undergoing embryo reduction to dichorionic twins by intrafetal laser

\begin{tabular}{|c|c|c|c|c|c|}
\hline \multicolumn{2}{|l|}{ Embryo reduction } & \multicolumn{2}{|c|}{ Separate fetus } & \multicolumn{2}{|c|}{ Monochorionic co-twin } \\
\hline findings in selected fetus & GA, weeks & outcome & GA, weeks & outcome & GA, weeks \\
\hline Donor in TTTS or selective FGR & 11.4 & live birth & 39.0 & fetal death & 12.0 \\
\hline Donor in TTTS or selective FGR & 11.4 & live birth & 39.4 & fetal death & 12.0 \\
\hline Donor in TTTS or selective FGR & 11.5 & live birth & 39.5 & fetal death & 12.1 \\
\hline None & 12.3 & live birth & 32.2 & fetal death & 12.5 \\
\hline None & 11.2 & live birth & 37.5 & fetal death & 13.0 \\
\hline None & 11.5 & live birth & 40.5 & fetal death & 13.0 \\
\hline None & 11.6 & live birth & 34.5 & fetal death & 13.0 \\
\hline None & 13.1 & live birth & 38.0 & fetal death & 14.0 \\
\hline Hydrops fetalis & 13.1 & live birth & 39.0 & fetal death & 14.1 \\
\hline None & 12.6 & live birth & 37.3 & fetal death & 14.4 \\
\hline Donor in TTTS or selective FGR & 13.0 & live birth & 34.4 & fetal death & 14.5 \\
\hline None & 13.2 & miscarriage & 23.9 & miscarriage & 23.9 \\
\hline None & 13.9 & live birth & 32.2 & live birth & 32.2 \\
\hline Donor in TTTS or selective FGR & 12.0 & live birth & 33.1 & live birth & 33.1 \\
\hline Donor in TTTS or selective FGR & 13.5 & live birth & 33.2 & live birth & 33.2 \\
\hline None & 11.1 & live birth & 34.4 & live birth & 34.4 \\
\hline None & 12.3 & live birth & 35.0 & live birth & 35.0 \\
\hline Donor in TTTS or selective FGR & 13.1 & live birth & 35.0 & live birth & 35.0 \\
\hline Diaphragmatic hernia & 12.6 & live birth & 36.1 & live birth & 36.1 \\
\hline None & 12.1 & live birth & 36.2 & live birth & 36.2 \\
\hline Donor in TTTS or selective FGR & 12.0 & live birth & 37.1 & live birth & 37.1 \\
\hline None & 12.2 & live birth & 37.1 & live birth & 37.1 \\
\hline
\end{tabular}

GA = Gestational age; TTTS = twin-to-twin transfusion syndrome; FGR = fetal growth restriction .

can be reduced by limiting the number of embryo transfers, the rate of monozygotic twinning is likely to increase because of the widespread use of blastocyst transfer and/ or zona manipulation, which may be the underlying causes for embryo splitting.

In the DCT pregnancies undergoing ER to dichorionic twins by intrafetal laser the rates of miscarriage and preterm birth $<33$ weeks were about 5 and $10 \%$, respectively. Although the number of cases presented in this study is too small for definite conclusions to be drawn, it appears that ER to dichorionic twins by intrafetal laser may be associated with a better outcome than with alternative management options [1]. We have recently reported that in DCT pregnancies expectant management is associated with high rates of both miscarriage (8\%) and early preterm birth (46\%), which could, at least in part, be attributed to complications arising from intertwin placental vascular communications and/or unequal sharing of the placenta in the monochorionic pair $[1,9]$. The option of ER of the separate fetus by intracardiac injection of $\mathrm{KCl}$ is associated with halving of the rate of preterm birth to $23 \%$, but an increase in the rate of miscarriage to $13 \%$ because the complications of monochorionic twins persist with the addition of complications arising from the intra-uterine presence of dead fetoplacental tissue $[1,9,10]$. The option of ER of the monochorionic pair with survival of the separate fetus results in a $14 \%$ rate of miscarriage, presumably due to the intra-uterine presence of excessive dead fetoplacental tissue, but a decrease in preterm birth to $8 \%$ [1]

In trichorionic triplet pregnancies management options include expectant, ER to twins and ER to singleton with respective rates of miscarriage of 3,7 and $12 \%$ and early preterm birth rates of 35, 13 and 9\% [1]. In the DCT pregnancies undergoing ER to dichorionic twins by intrafetal laser the rates of miscarriage and early preterm birth are similar to those in trichorionic triplet pregnancies undergoing ER to twins; however, in half of the cases only one of the babies survives.

\section{Acknowledgement}

This study was supported by grants from the Fetal Medicine Foundation (Charity No. 1037116). 


\section{References}

$>1$ Chaveeva P, Kosinski P, Puglia D, Poon LC, Nicolaides KH: Trichorionic and dichorionic triplet pregnancies at 10-14 weeks: outcome after embryo reduction compared to expectant management. Fetal Diagn Ther 2013;34: 199-205.

-2 Sepulveda W, Sebire NJ, Odibo A, Psarra A, Nicolaides KH: Prenatal determination of chorionicity in triplet pregnancy by ultrasonographic examination of the ipsilon zone. Obstet Gynecol 1996;88:855-858.

3 Robinson HP, Fleming JE: A critical evaluation of sonar crown rump length measurements. Br J Obstet Gynaecol 1975;82:702710.
4 O’Donoghue K, Barigye O, Pasquini L, Chappell L, Wimalasundera RC, Fisk NM: Interstitial laser therapy for fetal reduction in monochorionic multiple pregnancy: loss rate and association with aplasia cutis congenita. Prenat Diagn 2008;28:535-543.

5 Lewi L, Valencia C, Gonzalez E, Deprest J, Nicolaides KH: The outcome of twin reversed arterial perfusion sequence diagnosed in the first trimester. Am J Obstet Gynecol 2010;203: 213.e1-e4.

6 Scheier M, Molina FS: Outcome of twin reversed arterial perfusion sequence following treatment with interstitial laser: a retrospective study. Fetal Diagn Ther 2012;31:35-41.

$>7$ Vitthala S, Gelbaya TA, Brison DR, Fitzgerald CT, Nardo LG: The risk of monozygotic twins after assisted reproductive technology: a systematic review and meta-analysis. Hum Reprod Update 2009;15:45-55.
Aston KI, Peterson CM, Carrell DT: Monozygotic twinning associated with assisted reproductive technologies: a review. Reproduction 2008;136:377-386.

$\checkmark 9$ Sebire NJ, Snijders RJM, Hughes K, Sepulveda W, Nicolaides KH: The hidden mortality of monochorionic twin pregnancies. Br J Obstet Gynaecol 1997;104:1203-1207.

10 Abbas A, Johnson MR, Bersinger N, Nicolaides KH: Maternal alpha-fetoprotein levels in multiple pregnancies. Br J Obstet Gynaecol 1994;101:156-158. 